\title{
Trabalho na Atenção Básica: integralidade do cuidado em saúde mental
}

\author{
WORK IN PRIMARY HEALTH CARE: A COMPREHENSIVE MENTAL HEALTH CARE
}

\author{
TRABAJO EN LAATENCIÓN PRIMARIA DE SALUD: INTEGRALIDAD DEL CUIDADO EM \\ SALUD MENTAL
}

\author{
Juliana Reale Caçapava르, Luciana de Almeida Colvero², Wânia Regina Veiga Martines ${ }^{3}$, Ana Lúcia \\ Machado $^{4}$, Ana Luísa Aranha e Silva ${ }^{5}$, Divane de Vargas $^{6}$, Márcia Aparecida Ferreira de Oliveira ${ }^{7}$, \\ Sônia Barros ${ }^{8}$
}

\section{RESUMO}

Este estudo tem por objetivo cartografar o cuidado ao usuário com necessidades no campo da saúde mental em uma Unidade Básica de Saúde, analisando o trabalho em equipe à luz da integralidade das ações de saúde. Seus participantes são trabalhadores de saúde, de diferentes profissões, que fazem parte dos processos de trabalho em saúde mental do serviço. A técnica de coleta de dados utilizada foi o fluxograma analisador. Os resultados nos mostram que, na Unidade Básica, os fluxos conectivos entre os diversos trabalhadores - e entre estes e os usuários - vêm produzindo e proliferando vários e distintos espaços coletivos de trocas, possibilitando ações de saúde alinhadas à perspectiva da integralidade, através de uma compreensão ampliada do processo saúde-doença mental, construída pela valorização das relações humanas e das subjetividades envolvidas no espaço do trabalho em saúde.

\section{DESCRITORES}

Saúde mental.

Equipe de assistência ao paciente.

Atenção primária à saúde.

Assistência integral à saúde.

Cartografia.

\begin{abstract}
This study intends to map the health care given to the usuary with needs in the mental health field in a primary health care unity, analyzing the team work in the light of the integrate primary health care practices. The participants are health workers from different professions, who belong to the service's mental health working processes. The data collection technique used was the analytic flowchart. The results show us that the connective flows among the professionals and between them and the health unity's, in the primary health care unit, has been producing and proliferated diverse exchanges' spaces, making possible health actions aligned to the integrality in people's care through a enlarged understanding of health mental disease process, by means of human relationships' valuation and subjectivities involved in the health work space.
\end{abstract}

\author{
KEY WORDS \\ Mental health. \\ Patient care team. \\ Primary health care. \\ Comprehensive health care. \\ Cartography.
}

\begin{abstract}
RESUMEN
Este estúdio de abordaje cualitativa tiene como objetivo cartografiar el cuidado al usuário con necessidades en lo campo de la salud mental en uma Unidad Básica de Salud, a través de la análisis del trabajo en equipo a la luz de la integralidad de las acciones de salud. Sus partipantes son trabajadores de salud mental, de diferentes profesiones, que son parte de los procesos de trabajo en salud mental de lo servicio. La colección de datos fue construida mediante lo fluxograma analizador. Los resultados muestran que, en la Unidad Básica, los flujos conectivos entre los diversos trabajadores - $y$ entre estes y los usuarios - están a producir y proliferar vários y distintos espacios colectivos de intercâmbios, permitiendo acciones integrales de salud a través de una comprensión ampliada del proceso de salud-enfermedad mental, construida por médio de la valorización de las relaciones humanas y de las subjetividades involucradas en lo espacio del trabajo en salud.
\end{abstract}

\author{
DESCRIPTORES \\ Salud mental. \\ Grupo de atención al paciente. \\ Atención primaria de salud. \\ Atención integral de salud. \\ Cartografia.
}

\footnotetext{
${ }^{1}$ Enfermeira. Mestre em Enfermagem Psiquiátrica. Especialista em Laboratório do Departamento de Enfermagem Materno-Infantil e Psiquiátrica da Escola de Enfermagem da Universidade de São Paulo. São Paulo, SP, Brasil. jureale@uol.com.br ${ }^{2}$ Enfermeira. Professora Doutora do Departamento de Enfermagem Materno-Infantil e Psiquiátrica da Escola de Enfermagem da Universidade de São Paulo. São Paulo, SP, Brasil. lucix@usp.br ${ }^{3}$ Enfermeira. Doutoranda do Departamento de Enfermagem Materno-Infantil e Psiquiátrica da Escola de Enfermagem da Universidade de São Paulo. São Paulo, SP, Brasil. wanregina@terra.com.br ${ }^{4}$ Enfermeira. Professora Associada do Departamento de Enfermagem Materno-Infantil e Psiquiátrica da Escola de Enfermagem da Universidade de São Paulo. São Paulo, SP, Brasil. almachad@usp.br ${ }^{5}$ Enfermeira. Professora Doutora do Departamento de Enfermagem Materno-Infantil e Psiquiátrica da Escola de Enfermagem da Universidade de São Paulo. São Paulo, SP, Brasil. anaranha@usp.br ${ }^{6}$ Enfermeiro. Professor Doutor do Departamento de Enfermagem Materno-Infantil e Psiquiátrica da Escola de Enfermagem da Universidade de São Paulo. São Paulo, SP, Brasil. vargas@usp.br ${ }^{7}$ Enfermeira. Professora Associada do Departamento de Enfermagem Materno-Infantil e Psiquiátrica da Escola de Enfermagem da Universidade de São Paulo. São Paulo, SP, Brasil. marciaap@usp.br ${ }^{8}$ Enfermeira. Professora Associada do Departamento de Enfermagem Materno-Infantil e Psiquiátrica da Escola de Enfermagem da Universidade de São Paulo. São Paulo, SP, Brasil. sobarros@usp.br
} 


\section{INTRODUÇÃO}

Estudos focalizados na temática da saúde mental na Atenção Básica nos mostram que, embora os trabalhadores das Unidades Básicas de Saúde (UBS) reconheçam os fatores sociais como determinantes do processo saúdedoença da população, observa-se que o objeto do trabaIho é a doença mental, e, a partir deste objeto, o estabelecimento de diagnósticos e a prescrição de medicamentos é o meio ou instrumento de trabalho mais valorizado para a intervenção junto às pessoas com necessidades no campo da saúde mental ${ }^{(1)}$.

Acerca dessa discussão, é possível observar que a integralidade do cuidado precisa ser trabalhada em dimensões diferentes para ser alcançada da forma mais completa possível. Sendo assim, surgem as noções de integralidade focalizada e integralidade ampliada ${ }^{(2)}$.

A integralidade ampliada diz respeito à articulação de cada serviço de saúde a uma rede mais complexa, composta por outros serviços de saúde e outras instituições não necessariamente do setor saúde; ou seja, a integralidade pensada no macro. Já a integralidade focalizada é definida como o fruto do esforço e da confluência dos vários saberes de uma equipe multiprofissional, no espaço concreto e singular dos serviços de saúde ${ }^{(2)}$.

A articulação das ações de saúde, nos serviços, requer que o profissional reconheça e coloque em evidência as conexões e os nexos existentes entre as intervenções realizadas, sejam estas referidas tanto ao seu próprio processo de trabalho quanto às ações executadas pelos demais integrantes da equipe ${ }^{(3)}$.

Em face dessas premissas, no presente estudo nos propomos a aprofundar a compreensão da micropolítica de um serviço de saúde, por meio do agir cotidiano dos sujeitos, na relação entre si e no cenário em que eles se encontram ${ }^{(4)}$.

\section{OBJETIVO}

Consiste em cartografar o cuidado ao usuário com necessidades no campo da saúde mental em uma UBS, analisando o trabalho em equipe à luz da integralidade das ações de saúde.

\section{MÉTODO}

Optou-se pela pesquisa qualitativa, a qual possui a capacidade de incorporar a questão do significado e da intencionalidade como inerente aos atos, às relações e às estruturas sociais, estas últimas tomadas em seu advento e sua transformação, como construções humanas que podem ser apreendidas através do cotidiano e da vivência ${ }^{(5)}$.

O cenário do estudo é uma UBS do município de São Paulo, também considerada um Centro de Práticas Complementares, oferecendo à população atendimento em Medicina Tradicional Chinesa e Homeopatia. A coleta de dados deu-se a partir da construção coletiva do fluxograma analisador, com a participação de trabalhadores de saúde, que atuam nos processos de trabalho em saúde mental do serviço: psicólogo (dois); auxiliar de enfermagem (um); médico (três); assistente social (um). Foram dois encontros, cada qual com duração aproximada de 90 minutos.

O fluxograma analisador pode ser definido como um diagrama utilizado para desenhar todas as etapas do processo de trabalho, a partir da trajetória do usuário no serviço, a saber: a entrada ou saída do processo de produção de serviços (representada pela elipse); os momentos de decisão para a continuidade do trabalho (representada pelo losango) e o momento de intervenção, ação sobre o processo (representados pelo retângulo) ${ }^{(6)}$.

Para isso, foi proposto que os trabalhadores elegessem um usuário típico sobre o qual se debruçariam para descrever a trajetória do mesmo no serviço, solicitando a eles que, nesta escolha, fossem levadas em conta algumas diretrizes propostas: estar há pelo menos seis meses freqüentando o serviço, o que possibilita um retrato mais condizente com a realidade de seu cotidiano de trabalho; ter passado pelo menos por três profissionais do serviço, para avaliar a integração das ações entre os profissionais e relações do serviço com o usuário(7). Aproveitamos para ressaltar que todos os nomes aqui mencionados são fictícios.

O trabalho foi submetido à apreciação do Comitê de Ética em Pesquisa da Secretaria de Saúde do Município de São Paulo, do qual obtivemos aprovação por meio do parecer $n$ ㅇ 283/07.

\section{RESULTADOS}

\section{Descrição do fluxograma}

Os trabalhadores escolheram o usuário Bernardo, que, segundo eles, deixou marcas por ter mobilizado toda a equipe multiprofissional.

A entrada de Bernardo ao serviço se deu a partir de sua mãe, Manuela, que busca a assistente social do serviço com uma demanda de orientação sobre onde conseguir medicamentos. No desenrolar da conversa, Manuela queixa-se estar deprimida, sendo encaminhada à psicóloga. No entanto, antes desta consulta, a assistente social procura pela colega, para discutirem o caso. Quando atendida pela última, esta convida Manuela a participar do 
Grupo de Psicoterapia (GP) da UBS, no qual a usuária fala pela primeira vez de Bernardo, dizendo, de acordo com a psicóloga, que tinha um filho com problema, porque ele apresentava os mesmos comportamentos de seu falecido marido, que era esquizofrênico e viveu internado por muitos anos; situação com a qual, segundo Manuela, fora muito complicado conviver.

No GP a mãe é então orientada a trazer seu filho ao serviço em uma das datas direcionadas ao acolhimento em saúde mental. Participando do acolhimento a Bernardo, que tinha 16 anos, a mesma psicóloga o encaminha ao Grupo de Adolescentes (GA).

Por conseguinte, Bernardo passa a participar do GA, enquanto sua mãe, Manuela, continuava no GP, e também se insere, convidada pela psicóloga, no Grupo de Orientação de Pais (GOP).

Num dado momento, os profissionais da equipe de saúde mental resolvem, por meio dessas discussões, face às suas articulações e interações, que Bernardo deveria passar também com a médica homeopata, integrante daquela equipe e que trabalhava com grupos de crianças e saúde escolar. Esta médica, então, sente necessidade de encaminhar Bernardo à psiquiatra do serviço de referência do território, que aqui nomearemos como Centro de Referência (CR).

Os profissionais de saúde responsáveis pelo GA também percebem que o usuário, por apresentar um comportamento bastante regredido, não conseguia se vincular ao mesmo, questão que ao ser discutida na reunião da equipe de saúde mental, é direcionada no sentido de encaminhar Bernardo ao Grupo de Crianças de 10 a 11 anos, o Grupo de Orientação Lúdica (GOL), o qual, segundo a psicóloga e a médica homeopata, suas coordenadoras, foi o grupo onde ele se deu melhor.

Por meio deste percurso, observa-se que foram se configurando redes de conversação (individuais e coletivas) que interligaram os trabalhadores da equipe de saúde mental, acolhendo tanto Bernardo, quanto Manuela no serviço, permeadas também por reuniões quinzenais da equipe de saúde mental e um fluxo de conversas não-formais entre os trabalhadores alimentando a potência dos usuários produzirem relações em diferentes espaços, na UBS.

Então, aconteceu que Bernardo, em virtude de um grave problema oftalmológico, foi levado pela mãe até um hospital geral, de onde os médicos o encaminham ao setor de psiquiatria. Neste local, é feito o diagnóstico de esquizofrenia para o adolescente, embora para os profissionais da equipe de saúde mental da UBS aquele fosse controverso, levandose em consideração, para além dos sinais e sintomas, especialmente o contexto familiar envolvido:

O rótulo de esquizofrênico a Bernardo foi dado por uma visão mais clínica da psiquiatria, que não tem muito essa coisa de leitura de família, é mais a psicopatologia (Gerânio).

E, a partir do hospital geral, Bernardo foi encaminhado ao CAPS, tendo permanecido concomitantemente na UBS por pouco tempo, cerca de um mês. Segundo a assistente social, Manuela continuava freqüentando o serviço, porque participava do GP, apesar de muito faltosa; mas, abandonara o GOP. Para os profissionais da UBS, em relação a Bernardo, essas vindas de Manuela, ainda que espaçadas, eram importantes, por serem espaços de conversação nos quais eles perguntavam sobre o adolescente, sendo uma forma de saber como ele estava caminhando na vida, como andava a relação entre mãe e filho...

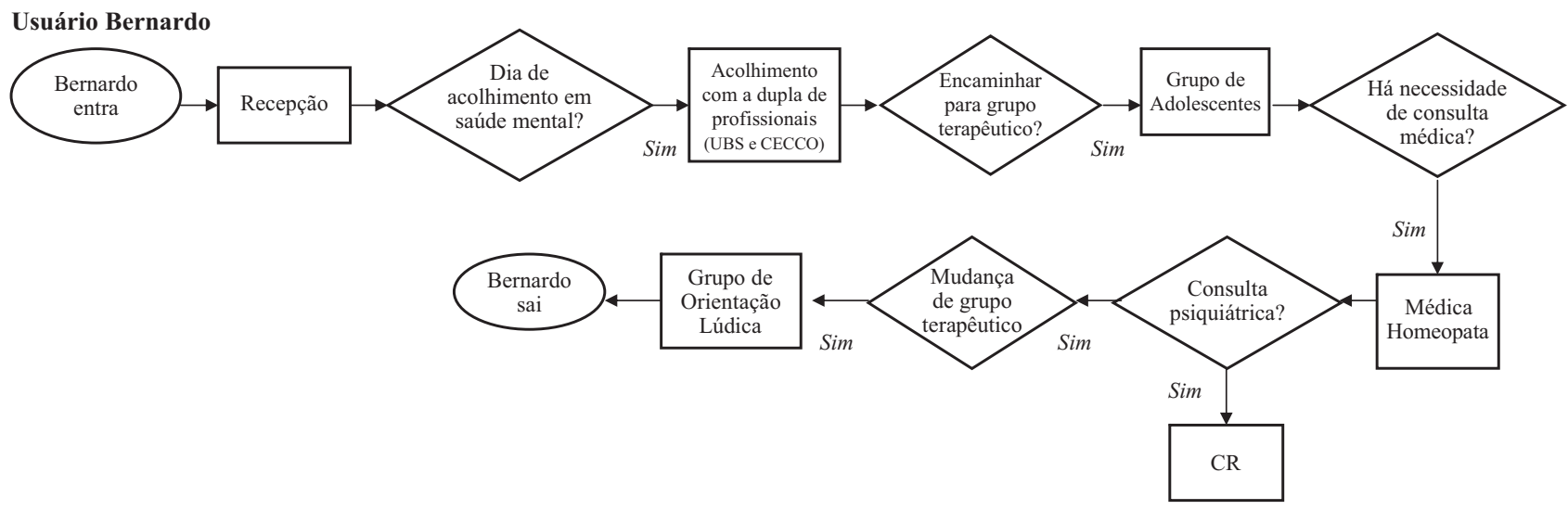

Figura 1 - Fluxograma analisador

\section{DISCUSSÃO}

A partir do que consideramos o primeiro acolhimento realizado pela UBS à Manuela, quando do seu encontro com a assistente social, pudemos observar que uma escuta qualificada e uma boa conversa podem ser dispositi- vos facilitadores do fluxo do usuário no serviço, pois, quando esta investiga as necessidades de Manuela, muito mais do que apenas a orientá-la quanto à questão dos medicamentos, que era sua demanda, aproxima-se de uma noção de cuidar que vai além do cuidado técnico, que implica importar-se com a pessoa, envolver-se com ela ${ }^{(8)}$. 
De acordo com a médica Tulipa, que é acupunturista, a experiência profissional na UBS foi lhe trazendo um olhar ampliado sobre a clínica, através do qual passou a entender as dores físicas como algo a explicitar dores psíquicas, exemplificando um caso em que uma paciente melhora consideravelmente quando a médica passa a doar-se mais à relação, através de uma escuta interessada que permitiu a ambas um insight de que o problema em questão relacionava-se à forma com a qual a usuária vinha enfrentando a sua vida.

Pudemos perceber que a rede de conversações que se formou no serviço sustentou-se pela interação e articulação entre os profissionais que, através de várias discussões, refletiam sobre seus modos de agir individual e coletivo e decidiam, a partir desses debates, os itinerários terapêuticos a serem experimentados, agenciando, portanto, singularidades e acolhendo-se uns aos outros. Assim, de acordo com Violeta, os trabalhadores, mesmo diante de situações difíceis (foram citados casos de abuso sexual, tentativa de suicídio) sabem que têm com quem dividir, têm um apoio que dá segurança para continuar...

Em relação à Manuela e Bernardo, destaca-se a forma como os profissionais buscaram interligar os dois casos, promovendo a integralidade do cuidado através de uma compreensão mais ampla do processo de sofrimento psíquico de ambos.

Com o fluxograma, pudemos notar a sensibilização e mobilização da equipe de saúde mental da UBS em relação ao caso, articulando-se e interagindo no sentido de buscar possibilidades de acolher Bernardo e vinculá-lo ao serviço, a despeito das referidas dificuldades em envolver Manuela na perspectiva da promoção da autonomia do adolescente frente à vida, dissuadindo-a de sua obstinação por um rótulo diagnóstico ao filho; tanto que este somente foi dado por outro serviço de saúde.

E, para Amarílis, a riqueza do trabalho desta equipe, em relação a este caso, esteve na disponibilidade dos profissionais e no espaço que foi aberto a eles, democrático e sob a égide de um agir coletivo, com potências para a invenção, porque foi o cuidado a Bernardo e a mais dois adolescentes, que disparou a criação do Grupo de Adolescentes na UBS. Assim, segundo Gerânio, a construção deste grupo se deu:

\section{[...] com muita discussão, 'entrando juntos' e tirando de nós aquele imediatismo em resolver as coisas, pensando em discutir o tipo de solução que a gente pode oferecer com o que a pessoa quer; foi aí que as coisas foram entrando nos eixos e a gente foi ficando com uma lingua- gem cada vez mais próxima [...].}

À época da coleta de dados, os profissionais da equipe de saúde mental da UBS relataram que sabiam por Manuela e Peônia (que trabalhava concomitantemente no serviço e no CAPS) da boa evolução de Bernardo; este participava de um grupo de adolescentes, no CAPS, e também estava namorando uma usuária, notícia considerada como sendo muito positiva, haja vista que uma das principais dificuldades que haviam sido apontadas, com relação ao usuário, dizia respeito à sua afetividade. Ademais, a assistente social comenta que Bernardo havia recebido o bilhete gratuito de transporte público, e que passara a se locomover de ônibus sozinho, pelas imediações do bairro.

Então, pergunta-se a eles sobre como esse atendimento havia os afetado, enquanto trabalhadores, e como, em sua opinião, havia afetado os usuários, na maneira de ser, pensar, agir e sentir. Emergem alguns insights a respeito de seus processos de trabalho em equipe, na Unidade Básica, refletindo sobre o compartilhamento que realizam dos casos ao privilegiarem os espaços de discussão e de troca com dinamismo e sintonia.

É proposto aos trabalhadores que procurassem retomar a concepção de cada um sobre cuidado, e refletindo se proporiam mudanças nos processos de trabalho em saúde mental, na UBS, e, em caso afirmativo, quais seriam essas mudanças. Então percebemos que, a partir desta discussão, são verdadeiramente apontados os mapas dos conflitos enfrentados por esta equipe, na UBS, já que eles nos relatam que, infelizmente, não havia essa articulação e interação com os outros profissionais do serviço, que se negavam a trabalhar em grupo, manifestando isto inclusive nas reuniões técnicas.

Para Amarílis, essa impermeabilidade entre as diferentes categorias profissionais é muito ruim, porque, do contrário:

Quando há troca sai isso que vemos nesta equipe, são olhares diferentes, e quem ganha com isso é o paciente...

Nessa perspectiva, algumas questões podem ser levantadas acerca da importância de um serviço de saúde tornar-se um espaço confortável, participativo e satisfatório, já que esta seria uma forma de valorizar o trabalhador e fazer com que o trabalho possa ser libertador e criativo(9).

\section{CONSIDERAÇÕES FINAIS}

De acordo com os achados, a organização dos processos de trabalho em saúde mental engendra-se na UBS pelo trabalho de uma equipe que interage e se articula ao acoIher o usuário, ou seja, disparando múltiplas redes de conversa nos encontros promovidos entre esse usuário e outros trabalhadores, usuários, serviços e a comunidade; enfim, encontros entre territórios-vida, pautados pelo eixo da integralidade.

Em contrapartida, vimos que os trabalhadores percebem também, no cotidiano do trabalho, as relações que não possibilitam o encontro dos núcleos profissionais no sentido da articulação de saberes e fazeres para pro- 
duzir os projetos de intervenções em saúde - compreendendo-as então como capturantes do mundo subjetivo do usuário e de seu modo de representar e construir necessidades de saúde, porque não seria a partir desse modo de cuidar que o usuário entraria com o seu mundo complexo, de vontades, desejos, saberes.

Portanto, a construção coletiva do fluxograma mobilizou a discussão dos trabalhadores sobre relações afetivas entre os sujeitos envolvidos nos encontros realizados no serviço, sejam eles trabalhadores ou usuários, na pers-

\section{REFERÊNCIAS}

1. Caçapava JR, Colvero LA. Estratégias de atendimento em saúde mental nas Unidades Básicas de Saúde. Rev Gaúcha Enferm. 2008;29(4):573-80.

2. Cecílio LCO. As necessidades de saúde como Conceito Estruturante na Luta pela Integralidade e Equidade na Atenção em Saúde. In: Mattos RA, Pinheiro R, organizadores. Os sentidos da integralidade na atenção e no cuidado à saúde. Rio de Janeiro: IMS/UERJ- CEPESC/ ABRASCO; 2001. p. 113-50.

3. Peduzzi M. Trabalho em equipe de saúde no horizonte normativo da integralidade, do cuidado e da democratização das relações de trabalho. In: Pinheiro R, Barros de Barros ME, Mattos RA. Trabalho em equipe sob o eixo da integralidade: valores, saberes e práticas. Rio de Janeiro: CEPESC/ABRASCO; 2007. p. 161-78.

4. Franco TB. As Redes na Micropolítica do Processo de Trabalho em Saúde. In: Pinheiro R, Mattos RA, organizadores. Gestão em Redes: práticas de avaliação, formação e participação na saúde. Rio de Janeiro: CEPESC/ IMS/UERJ/ABRASCO; 2006. p. 459-74.

5. Minayo MCS. O desafio do conhecimento: a pesquisa qualitativa em saúde. São Paulo: Hucitec; 2004. pectiva de um olhar crítico-reflexivo sobre os espaços de conversação nos serviços de saúde.

Acreditamos, assim, que este estudo possibilitou à equipe experimentar e refletir para: evitar que os trabalhadores se fechem em suas concretudes resolutivas e potências; reinventar um cotidiano que transversalize também a organização dos processos de trabalho dos profissionais resistentes, de modo que estes ousem ao gerar novas possibilidades anti-hegemônicas na compreensão da multiplicidade do sofrimento psíquico do usuário.

6. Franco TB. Fluxograma descritor e projetos terapêuticos para análise de serviços de saúde, em apoio ao planejamento: o caso de Luz (MG). In: Merhy EE, Magalhães Júnior HM, Rimoli J, Franco TB, Bueno WS. O trabalho em saúde: olhado e experienciando o SUS no cotidiano. São Paulo: Hucitec; 2003. p. 161-98.

7. Carvalho LC, Merhy EE, Silva Júnior AG. Refletindo sobre o ato de cuidar da Saúde. In: Mattos RA, Pinheiro R, organizadores. Construção da integralidade: cotidiano, saberes e práticas em saúde. Rio de Janeiro: IMS/UERJ/ ABRASCO; 2003. p. 115-30.

8. Sadala MLA, Silva FM. Cuidando de pacientes em fase terminal: a perspectiva de alunos de enfermagem. Rev Esc Enferm USP. 2009;43(2):287-94.

9. Santos-Filho SB. Dando visibilidade à voz dos trabalhadores: possíveis pontos para uma pauta de valorização? In: Santos-Filho SB, Barros de Barros ME, organizadores. Trabalhador de saúde, muito prazer!: protagonismo dos trabalhadores na gestão do trabalho em saúde. Ijuí: Ed. Unijuí; 2007. p. 261-6. 